\title{
Study on the Response and Recovery Characteristics of Different Herbaceous Peony (Paeonia lactiflora Pall.) Varieties to Waterlogging Stress
}

\author{
Qingxia Zhang1, Dongliang Zhang', Anqi Xie', Jinguang $\mathrm{Xu}^{2}$, \\ Xue Li1, Yang Li1, Zemiao Liu' ${ }^{1}$, Xia Sun ${ }^{1 *}$ \\ ${ }^{1}$ College of Horticulture, Shandong Agricultural University, State Key Laboratory of Crop Biology, Taian, China \\ ${ }^{2}$ Shandong Academy of Forestry, Jinan, China \\ Email: 1539694800@qq.com, *guanshanghuayuan@163.com, sunxia65@sina.com
}

How to cite this paper: Zhang, Q.X., Zhang, D.L., Xie, A.Q., Xu, J.G., Li, X., Li, Y., Liu, Z.M. and Sun, X. (2021) Study on the Response and Recovery Characteristics of Different Herbaceous Peony (Paeonia lactiflora Pall.) Varieties to Waterlogging Stress. American Journal of Plant Sciences, 12, 1361-1379.

https://doi.org/10.4236/ajps.2021.129096

Received: July 30, 2021

Accepted: September 13, 2021

Published: September 16, 2021

Copyright $\odot 2021$ by author(s) and Scientific Research Publishing Inc. This work is licensed under the Creative Commons Attribution International License (CC BY 4.0).

http://creativecommons.org/licenses/by/4.0/

\begin{abstract}
The root of herbaceous peony (Paeonia lactiflora Pall.) is fleshy, and different varieties have different tolerance to waterlogging stress. In order to explore its response and recovery characteristics to waterlogging stress, six varieties of herbaceous peony with strong, medium and weak waterlogging tolerance and high ornamental value were selected as experimental materials. After the vegetative growth of each variety was completed, the field simulated waterlogging stress experiment was carried out by the semi-flooded (the water surface is half the height of the flowerpot) pot method. Changes in photosynthetic parameters, chlorophyll fluorescence parameters, relative water content (RWC), relative conductivity (REC), chlorophyll content, lutein cycle, and leaf microstructure were analyzed during recovery from waterlogging stress and stress relief. The results showed that the time of reaching the most significant difference between $\mathrm{CK}$ and tested varieties was different. From the beginning of stress to $60 \%$ of the leaves with symptoms, the varieties with strong and moderate waterlogging tolerance experienced longer time; the proportion of palisade tissue in leaves was larger; the maximum photochemical quantum yield (Fv/Fm) was reduced less; it could increase xanthophyll cycle and heat dissipation (NPQ) to consume excess light energy, and maintain a higher net photosynthetic rate $(\mathrm{Pn})$ for normal growth of plants in a short period of time. The REC in leaves of varieties with weak waterlogging tolerance increased more, and the damage of cell membrane was more serious. After the stress was removed, all indexes recovered to different degrees. Based on our comprehensive analysis, the comprehensive waterlogging resistance of the expe-
\end{abstract}


rimental materials followed the pattern: "Lihong" > "Yangfeichuyu" > "Taohuafeixue" > "Dafugui" > "Qihualushuang" > "Hongxiuqiu". It is suggested that the variety "Lihong" and "Yangfeichuyu", with strong comprehensive waterlogging tolerance, can be selected for propagation and cultivation in areas prone to waterlogging. In the later stage, it is still necessary to further expand the number and scale of varieties, combined with the in-depth study of waterlogging-resistance genes, so as to provide a theoretical reference for the cultivation and production of new waterlogging-resistant varieties.

\section{Keywords}

Herbaceous Peony, Waterlogging, Photosynthesis, Chlorophyll Fluorescence, Structure Microhumid, Lutein Cycle

\section{Introduction}

Waterlogging can lead to a decrease in soil oxygen content; change the physiological and biochemical properties of soil; hinder the aerobic respiration and absorption of nutrients by plants; reduce the net photosynthetic rate, stomatal conductance, and transpiration rate of leaves; reduce the chlorophyll content; significantly reduce the apparent quantum efficiency and the maximum electron transfer rate of leaves; and reduce the leaf area and membrane stability of plants [1] [2]. Together, these responses have a significant negative impact on the morphological characteristics, growth, and development of plants, hindering the formation and development of new leaves, with initially red and yellow leaves, further wilting, rewinding, drooping, disease, and other symptoms, and accelerated abscission [3]. In response to damage to the photosynthetic system of leaves, plants have evolved mechanisms of light energy dissipation to prevent damage to their photosynthetic organs in response to excess light energy. These include dissipation of radiation energy through the xanthophyll cycle, which is one of the main mechanisms evolved by plants to resist light damage [4].

Herbaceous peony (Paeonia lactiflora Pall.) is a perennial herbaceous plant belonging to the Paeonia group in the Paeonia family. It is a famous traditional flower in China and has the name of "flower phase". It exists in a variety of colors and patterns, has a high ornamental value [5], and is widely used in gardens, traditional Chinese medicine, and as fresh cut flowers. During the growth of herbaceous peony, photosynthetic organic matter is stored in the root, forming a nutrient-rich expanded ratoon for the development of winter buds and the consumption of next spring flowering [6]. Excessive water induces early aging in leaves, weakens photosynthesis, and reduces organic matter accumulation, impacting the flowering quality in the following year. And it shows that short-term waterlogging caused the leaves of herbaceous peony to turn yellow or red and the roots to turn black [7]. Previous studies have investigated the response of herbaceous peony under drought stress [8], while few studies have focused on water- 
logging stress. However, different varieties of herbaceous peony had different tolerance to waterlogging. The purpose of this study was to analyze the different responses of peony varieties under waterlogging stress by simulating waterlogging stress, and to screen more waterlogging-resistant varieties, so as to provide a reference for the selection of waterlogging-resistant varieties for the high-efficiency, high-quality, and high-yield cultivation of peony.

\section{Material and Methods}

\subsection{Plant Materials and Experimental Treatments}

Based on a preliminary experiment, six varieties of herbaceous peony (Paeonia lactiflora Pall.) with strong, medium and weak waterlogging resistance and high ornamental value were selected: "Lihong", "Dafugui", "Hongxiuqiu”, "Qihualushuang", "Taohuafeixue", and "Yangfeichuyu". Three-year-old ramets, healthy and disease-free, with strong growth were selected as experimental materials. After the vegetative growth of each variety was completed, field experiments were carried out (the cultivation substrate was garden soil).

The experiment was carried out from April 2019 to April 2020 at the Garden Center Laboratory of Horticultural Experiment Station of Shandong Agricultural University, the Gardening Center Laboratory and the Life Science Center, the Herbaceous Peony of the Horticulture Test Station and the Experimental Center of the Academy of Life Sciences. It is located between $116^{\circ} 02^{\prime} \mathrm{E}$ to $117^{\circ} 59^{\prime} \mathrm{E}$ and $35^{\circ} 38^{\prime} \mathrm{N}$ to $36^{\circ} 28^{\prime} \mathrm{N}$, and is a temperate semi humid continental monsoon climate.

The experiment was divided into a waterlogging group (WL) and a control check group (CK), each treatment was repeated 3 times, 20 pots were used for sampling, and 5 pots were used for morphological observation. Waterlogging stress was removed when $60 \%$ (more than $60 \%$ is difficult to recover) of the leaves showed symptoms of injury. Waterlogging stress stage: It starts from the beginning of waterlogging stress treatment and ends when $60 \%$ of the leaves of each variety have symptoms of damage. Recovery stage: It begins when waterlogging stress is removed and ends when the leaves return to green (if it has not recovered in 18 days, it means that it cannot return to normal.). The WL used the "double-set basin" semi-flood method. Put the potted peony in a basin and keep the water level at $1 / 2$ of the flowerpot to simulate waterlogging stress (flowerpot specification: $30 \mathrm{~cm}$ high, $33 \mathrm{~cm}$ upper diameter, and $27 \mathrm{~cm}$ lower diameter; basin specification: $41 \mathrm{~cm}$ long, $33 \mathrm{~cm}$ wide, and $20 \mathrm{~cm}$ high). Water the potted plants daily to keep the soil water supersaturated. The spacing of potted plants is $10 \mathrm{~cm}$. The reed curtains are covered on the surface of the pot to prevent the sun from increasing the water temperature in the pot. CK is managed according to conventional planting.

Photographs were taken every day to record growth status. Samples were taken every 3 days. Photosynthetic and chlorophyll fluorescence indices were measured every 6 days. Calculations of waterlogging tolerance and index screening formu- 
las: The waterlogging stress phase is based on the stress time experienced by the earliest variety that was removed from the waterlogging stress, and the recovery phase is based on the time required for the earliest variety to be restored after the stress is removed. The index values of the tested varieties at the same time interval were selected to calculate and compare the waterlogging tolerance and recovery coefficients among varieties.

\subsection{Quantification of Chlorophyll Content, Relative Water Content of Leaves, and Relative Conductivity of Leaves}

The chlorophyll content and relative conductivity of leaves were compared using the methods described by Zhao and Cang [9].

Remove the experimental materials from the main vein, weigh about $0.2 \mathrm{~g}$ of leaves into a $50 \mathrm{~mL}$ centrifuge tube, add $25 \mathrm{~mL}$ of $95 \%$ ethanol and seal it in sequence, extract at room temperature for $48-72 \mathrm{~h}$ under dark conditions, and subject the extract to UV754N ultraviolet-visible spectrophotometry Meter: Colorimetric comparison was performed at wavelengths of 665, 649, and $470 \mathrm{~nm}$ respectively. Calculate the concentration of chlorophyll a, b and carotenoid $(\mathrm{mg} / \mathrm{L})$ based on the OD value of each wavelength, and calculate the content of each pigment (expressed in $\mathrm{mg} / \mathrm{g}$ fresh weight) based on the calculated concentration.

$$
\begin{gathered}
\mathrm{Ca}=13.95 \mathrm{D}_{665}-688 \mathrm{D}_{649} ; \mathrm{Cb}=24.96 \mathrm{D}_{649}-732 \mathrm{D}_{665} ; \\
\mathrm{Cx}=1000 \mathrm{D}_{470}-205 \mathrm{Ca}-114.8 \mathrm{Cb} / 245 .
\end{gathered}
$$

The content of pigment in leaves $(\mathrm{mg} / \mathrm{g}=$ pigment concentration $(\mathrm{mg} / \mathrm{L}) \mathrm{x}$ total volume of extract $(\mathrm{mL}) \mathrm{x}$ dilution factor/sample mass $(\mathrm{g})$.

Wash the fresh leaves with deionized water, punch with a hole punch with a diameter of $1 \mathrm{~cm}$, take $0.1 \mathrm{~g}$ of the sample into a test tube, add $20 \mathrm{~mL}$ of deionized water, shake the shaker $\left(230 \mathrm{r} \cdot \mathrm{min}^{-1}\right)$, and determine the initial solution conductivity $\mathrm{C}_{1}$, And then boil the water bath for 30 minutes, measure the conductivity $\mathrm{C}_{2}$, and calculate $\mathrm{REC}=\mathrm{C}_{1} / \mathrm{C}_{2} \times 100 \%$ according to the formula.

The relative water content of the leaves was determined by the dry weighing method [10]. Cut the leaves, dry the surface, quickly weigh the fresh weight (FW), then immerse it in distilled water, place it in a dark place at $4^{\circ} \mathrm{C}$ for 24 hours to absorb water, take it out, dry the surface water with absorbent paper, and weigh the saturated fresh weight (SFW), put them in an oven at $105^{\circ} \mathrm{C}$ for 30 minutes, and dry them at $80^{\circ} \mathrm{C}$ for 10 hours to constant weight. Weigh the dry weight $(\mathrm{DW})$, and use the formula RWC $=(\mathrm{FW}-\mathrm{DW}) /(\mathrm{SFW}-\mathrm{DW}) \times 100 \%$ to calculate the relative moisture content.

\subsection{Quantification of Photosynthetic Gas Exchange and Chlorophyll Fluorescence Parameters}

Photosynthetic and chlorophyll fluorescence parameters were measured from 8:00 to 9:00 am on the sunny and windless days, using the $4^{\text {th }}$ to $5^{\text {th }}$ mature leaves from the parietal lobe. Photosynthetic gas exchange parameters were measured 
using a CIRAS-3 portable photosynthetic apparatus (PP Systems, USA). Chlorophyll fluorescence parameters were determined using a FMS-2 portable pulsemodulated fluorometer (Hansatech, UK).

\subsection{Quantification of Lutein Cycle}

Lutein-related indicators were quantified by HPLC. Conversion between the constituents of the lutein circulating component is expressed as $(\mathrm{A}+\mathrm{Z}) /(\mathrm{A}+\mathrm{Z}+\mathrm{V})$ [11], where, $A$ is the anther yellow matter content, $Z$ is the zeaxanthin content, and $\mathrm{V}$ is the violaxanthin content.

\subsection{Preparation of Paraffin Section of Leaf}

Samples were taken from leaves at three stages: stress initiation, stress relief,and final recovery. The materials were fixed with FAA fixative solution and paraffin sections were made with reference to a previously described method [12]. Then, samples were observed under a Motic BA300 microscope, photographed with Motic Images Advanced 3.2 and Moticam 2006 to compare their structural changes, and microstructural parameters of leaves were measured using Image-Pro plus software. (1) Cell tense ratio (CTR, \%) = the thickness of palisade organization/ the thickness of leaf $\times 100$; Spongy ratio (SR, \%) $=$ the thickness of sponge tissue/the thickness of leaf $\times 100$; Vein protuberant degree (VPD, \%) $=$ the thickness of the vein/the thickness of leaf $\times 100$

\subsection{Data Processing and Analysis}

Data were processed and mapped using Microsoft Excel software, and data were tested for significance using DPS (Duncan's new complex range method, P < 0.05). SPSS Statistics V 25.0 software was used for data principal component analysis, cluster analysis (membership function method) and stepwise regression analysis.

\section{Results}

\subsection{Effects of Waterlogging Stress on the Morphology and Microstructure of Six Herbaceous Peony Varieties}

As shown in Table 1 and Figure 1(a), the stress tolerance time of different varieties varies from the beginning of waterlogging stress to the time when $60 \%$ of leaves turn yellow or red. "Dafugui" has the longest time (27 days), "Hongxiuqiu" and "Qihualushuang" have the shortest time (6 days). After the removal of stress, the recovery time of "Dafugui" was the longest (18 days) and "Taohuafeixue" was the shortest ( 6 days). When the stress was removed, the cell gap of

Table 1. Treatment time of test materials.

\begin{tabular}{ccccccc}
\hline & “Lihong" & “Dafugui” & “Hongxiuqiu” & “Qihualushuang” & “Taohuafeixue” & "Yangfeichuyu” \\
\hline Waterlogging stress stage (days) & 14 & 27 & 6 & 6 & 12 & 10 \\
Recovery stage (days) & 12 & 18 & 9 & 9 & 6 & 9 \\
\hline
\end{tabular}


(a)
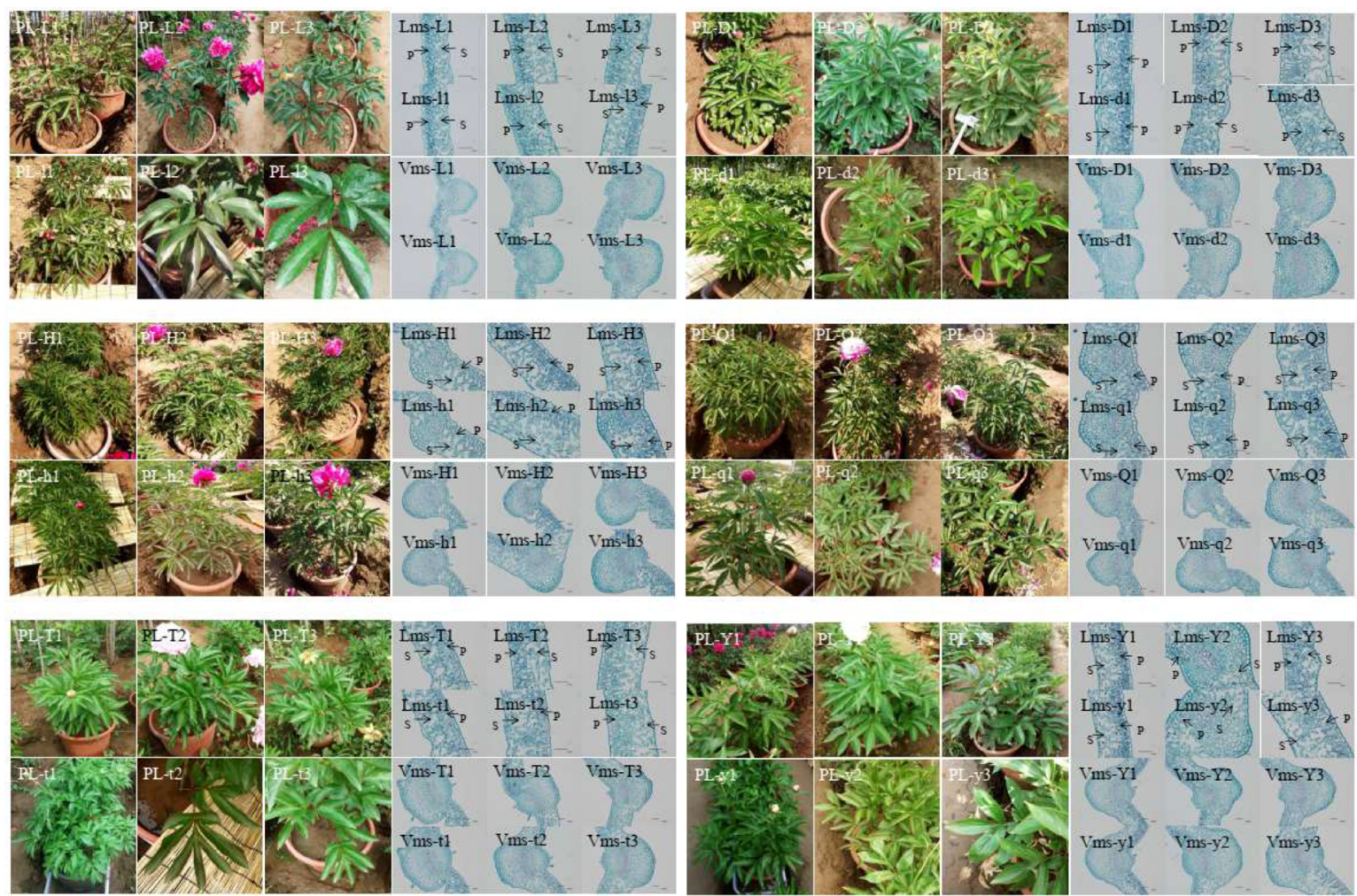

(b)

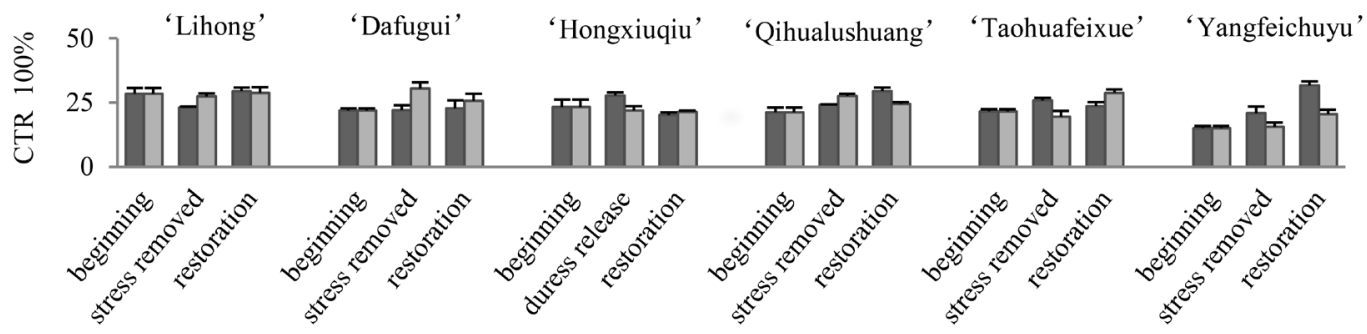

(c)

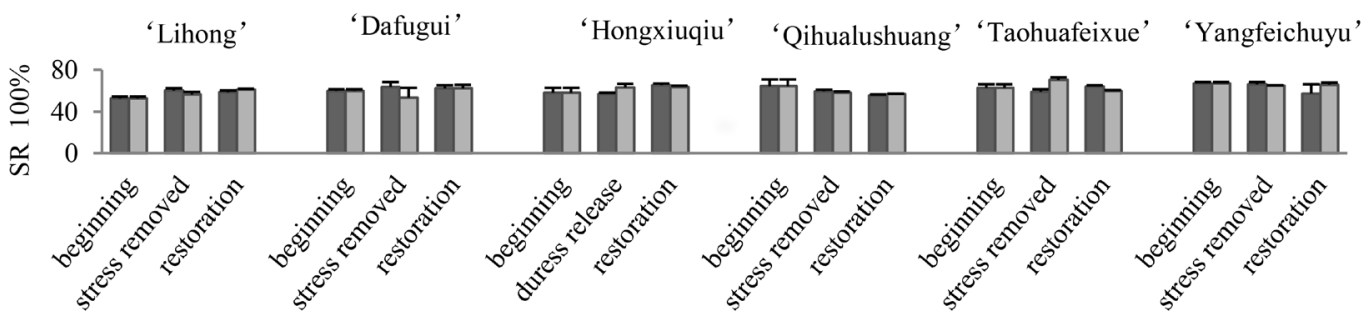

(d)

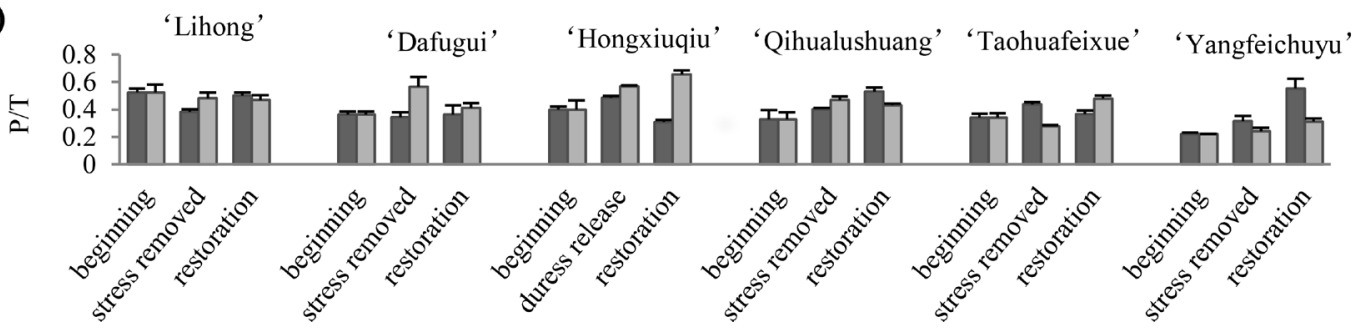


(e)

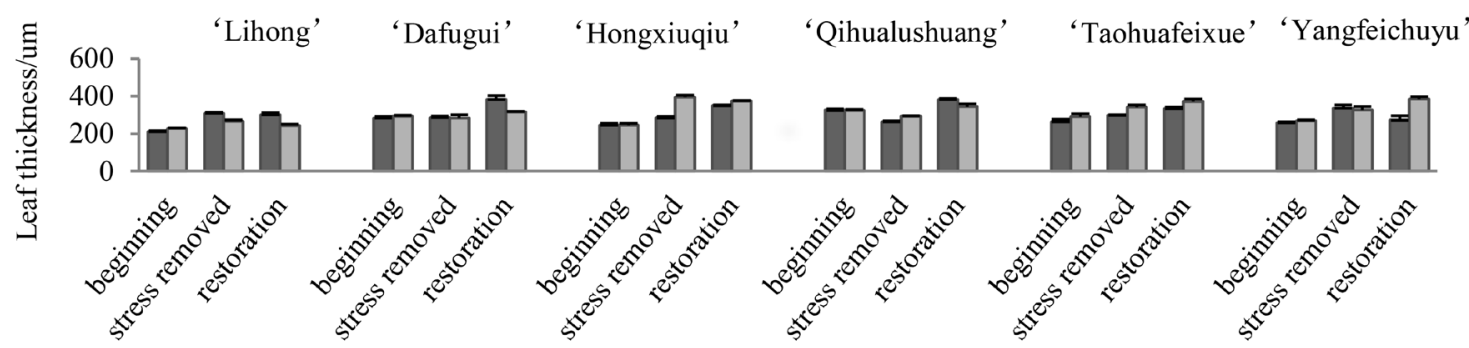

(f)

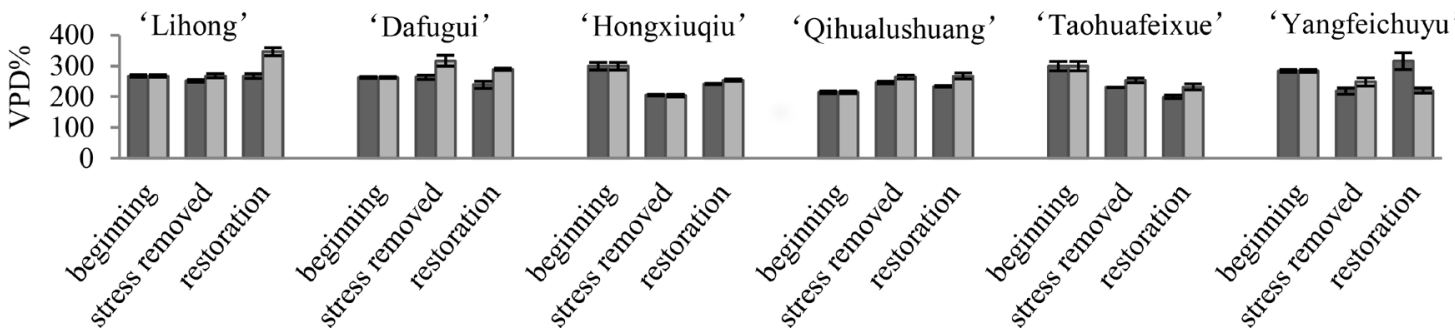

Figure 1. Morphology of the tested materials and changes in the microstructure of leaves. (a) P (palisade tissue); $S$ (sponge tissue); Lms (Leaf cross-section microstructure) $(\times 200)$; Vms (central vein transverse section microstructure) ( $\times 100) ; 1,2,3$ respectively represent the beginning of waterlogging stress, removal of stress and recovery; PL-L, PL-D, PL-H, PL-Q, PL-T, PL-Y respectively indicates the control group of "Lihong" "Dafugui" "Hongxiuqiu" "Qihualushuang" "Taohuafeixue" and "Yangfeichuyu", the corresponding lowercase letter indicates the waterlogging group; (b) the change of leaf tissue structure compactness (CTR); (c) the change of leaf tissue structure porosity (SR); (d) is the change of the ratio of palisade tissue to sponge tissue $(\mathrm{P} / \mathrm{T})$; (e) the change of leaf thickness. Error bars represent the standard error of mean $(n=3, P<0.05)$.

palisade tissue and sponge tissue of "Hongxiuqiu" increased significantly (Figure 1(a): Lms-H2, h2).

As shown in Figure 1(b) and Figure 1(c), compared with CK, the cell tense ratio (CTR) "Da fugui" increased the most (38.28\%) when stress was removed, while the CTR of "Yangfeichuyu" and "Taohuafeixue" decreased the most $(25.09 \%$ and $24.85 \%)$. During recovery, "Taohuafeixue" increased the most than CK (21.57\%), while "Yangfeichuyu" decreased the most than CK (35.12\%). The spongy ratio (SR) and CTR were negatively correlated. As shown in Figure 1(d), compared with $\mathrm{CK}$, the ratio of "Taohuafeixue" palise tissue/sponge tissue $(\mathrm{P} / \mathrm{T}) \mathrm{de}$ creased the most (37.22\%) and the ratio of "Da fugui" increased the most (64.31\%) when the stress was removed. When recovering, the P/T of "Hongxiuqiu" increased the most (112.06\%) and the P/T of "Yangfeichuyu" decreased the most (43.55\%) compared with CK.

As shown in Figure 1(e), compared with CK, when the stress was removed, the leaf thickness of "Lihong" decreased the most, while that of "Hongxiuqiu" increased the most. At the time of recovery, "Yangfeichuyu" increased by $49.73 \%$ compared with CK. Vein protuberant degree (VPD) also changed significantly (Figure 1(f)). Compared with CK, the "great wealth" increased the most (20.48\%) when the stress was removed, and the "red hydrangea" was slightly lower. During the recovery, "Lihong" and "Dafugui" increased the most (29.90 and 21.06\%), while "Yangfeichuyu" decreased the most (30.47\%). 


\subsection{Effects of Waterlogging Stress on Relative Water Content and Relative Conductivity in Six Herbaceous Peony Varieties}

According to Figure 2, the relative water content (RWC) of "Lihong" and "Yangfeichuyu" was significantly higher than that of $\mathrm{CK}$ when the stress was removed. When recovering, the RWC of "Dafugui", "Taohuafeixue" and "Lihong" were significantly higher than those of CK. Other varieties are basically consistent with CK. The relative electrical conductivity (REC) represents the permeability of the cytoplasmic membrane. The larger the REC is, the more tissue exudate is, the more permeable the plasma membrane is, and the deeper the damage to the plant is [13]. Compared with CK, the REC of "Taohuafeixue" increased the most (27.04\%) when the stress was removed, and the REC of "Yangfeichuyu" was slightly lower than CK. During recovery, the REC of "Taohuafeixue" increased significantly, $42.82 \%$ higher than CK.

\subsection{Effect of Waterlogging Stress on Chlorophyll Content in Six Herbaceous Peony Varieties}

Chlorophyll content is the direct manifestation of photosynthetic performance, nutritional status, and senescence in plant leaves. The external manifestation of waterlogging stress is the loss of green leaves, yellowing, and littering [14]. As shown in Figure 3, compared with CK, the Chla $+\mathrm{b}$ content in the leaves of

(a) 'Lihong'

$\longrightarrow$ RWC-CK - RWC-WL
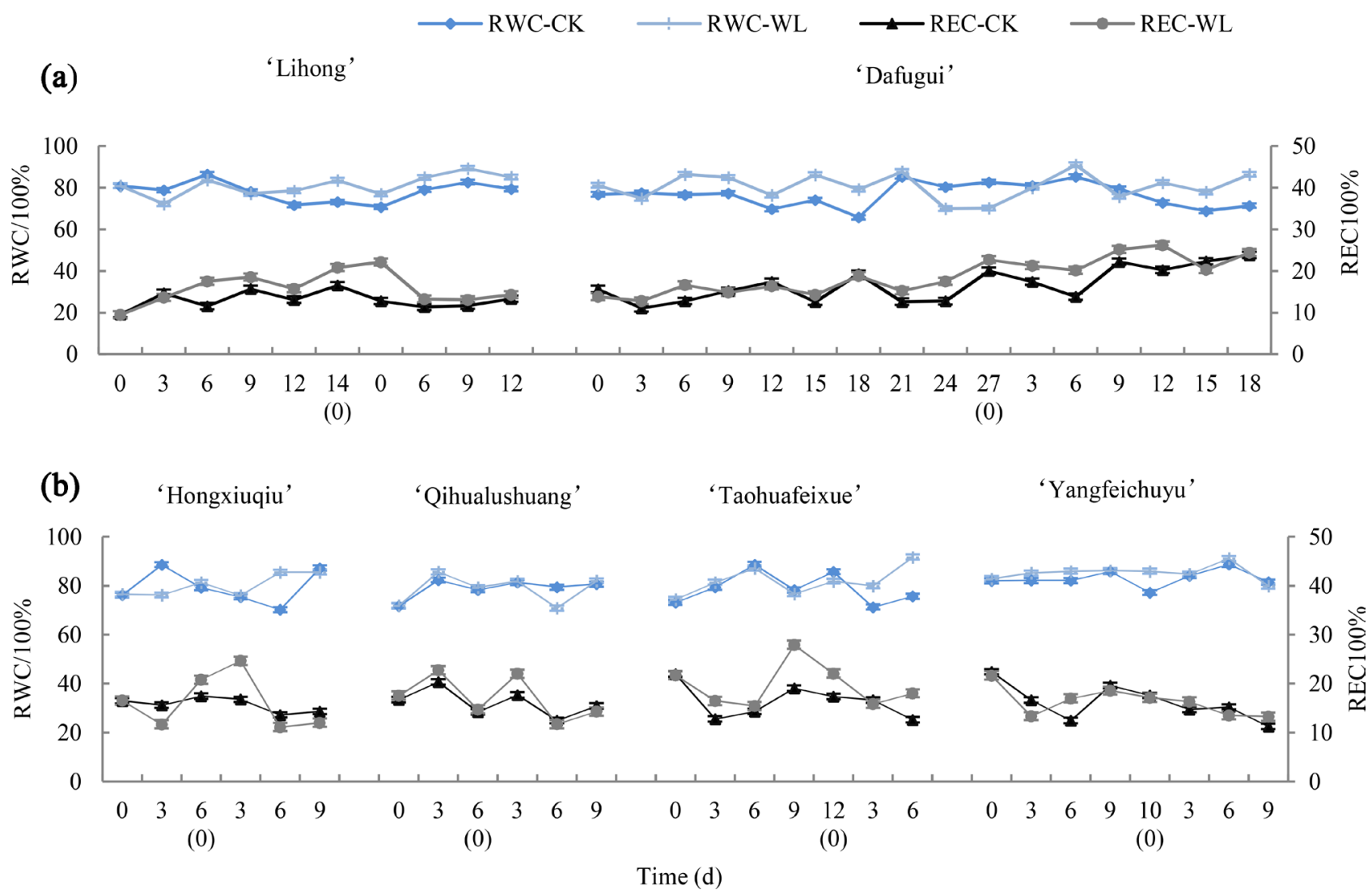

Figure 2. Changes in leaf relative water content (RWC) and electrical conductivity (REC) of test materials ((a), (b)). The error bars indicate standard error $(n=3)$. The time of stress removal is marked as the $0^{\text {th }}$ day of the recovery stage. 


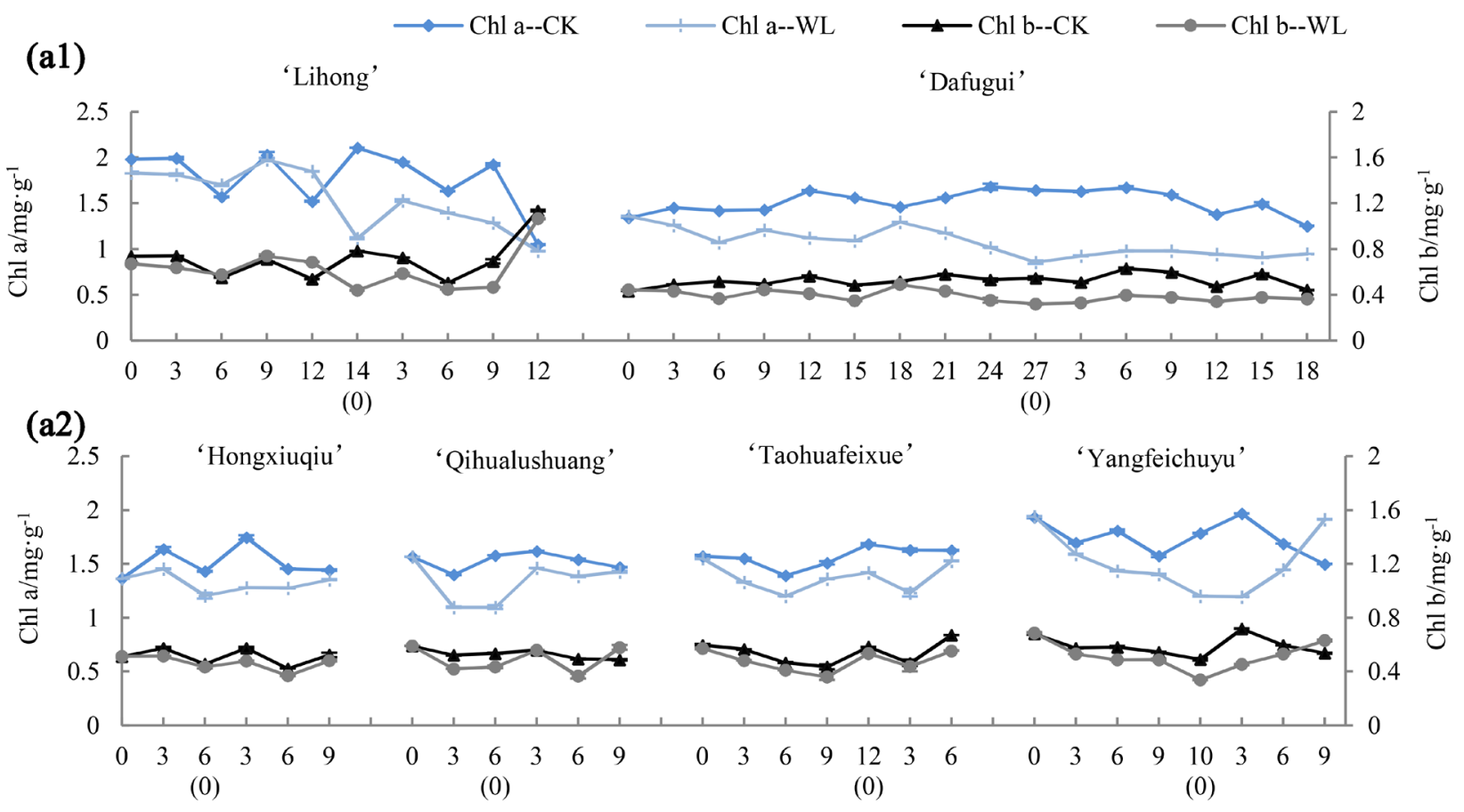

(b1)

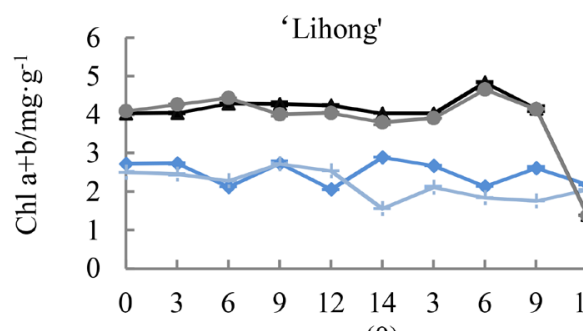

(0)

(b2)

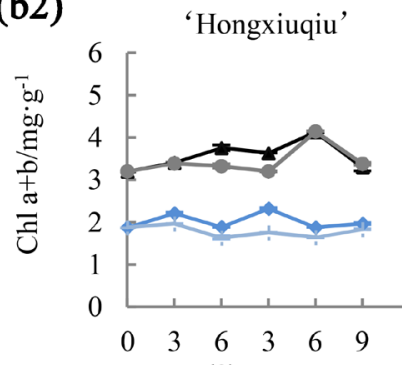

(0)

$\longrightarrow$ Chl a+b--CK

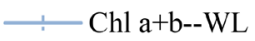

$\longrightarrow \mathrm{Chl} \mathrm{a/b--CK}$

$\longrightarrow \mathrm{Chl}$ a/b--WL

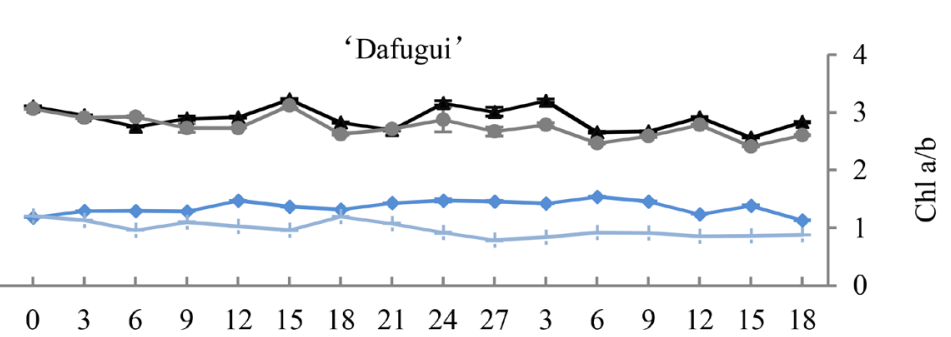

(0)

'Qihualushuang' ‘Taohuafeixue'

'Yangfeichuyu'

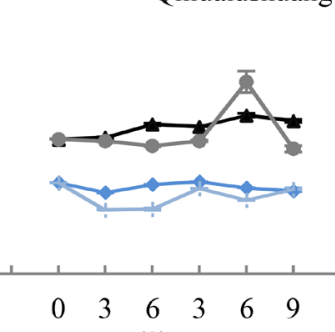

(0)
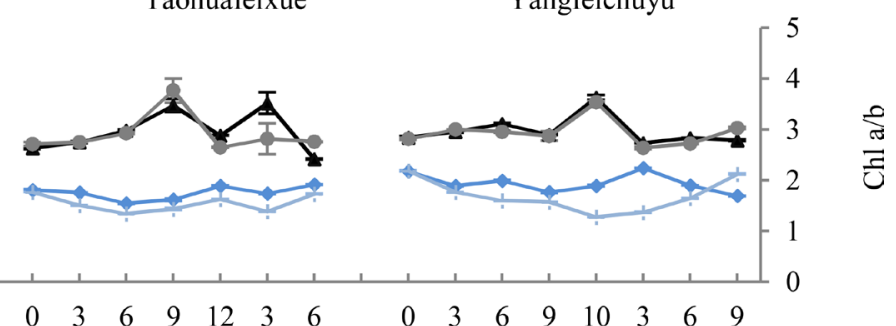

(0)

Time (d)

Figure 3. Changes of chlorophyll content in the tested materials. (a1), (a2): changes of chlorophyll A content and chlorophyll b content; (b1), (b2): change of total chlorophyll content and chlorophyll $\mathrm{a} / \mathrm{b}$. The error bars indicate standard error $(\mathrm{n}=3)$. The time of stress removal is marked as the $0^{\text {th }}$ day of the recovery stage.

"Lihong" and "Dafugui" showed the greatest difference when the stress was removed, with a decrease of $46.03 \%$ and $46.24 \%$, respectively. When recovering, "Lihong", "Hongciuqiu", "Qihualushuang", "Taohuafeixue" all recovered, "Da fugui" was lower than CK, "Yangfeichuyu" was higher than CK. The main function of $\mathrm{Chl} b$ in plant leaves is to collect light energy, while the main function of $\mathrm{Chl} \mathrm{a}$ is to convert the light energy collected by $\mathrm{Chl} \mathrm{b}$ into chemical energy. The 
variation trends of $\mathrm{Chl}$ a and $\mathrm{Chl} \mathrm{b}$ contents were consistent with that of $\mathrm{Chl} \mathrm{a}+$ b. In the early stage of stress, $\mathrm{Chl}$ a/b was basically unchanged compared with CK. When the stress was removed, Chl a/b was lower than CK, and Chl a content decreased more.

\subsection{Effects of Waterlogging Stress on Photosynthetic Gas Exchange Parameters in Six Herbaceous Peony Varieties}

As shown in Figure 4, following treatment, the photosynthetic rate (Pn), transpiration rate (E), and water utilization rate (WUE) of herbaceous peony varieties were significantly reduced by waterlogging stress. The Pn of "Lihong" was higher than CK in the 0 - 12 days of waterlogging stress, and increased $92.30 \%$ compared with CK on the $6^{\text {th }}$ day. When the stress was removed, "Lihong" and "Taohuafeixue" decreased the most than CK (94.05\% and 94.66\%). Compared with $\mathrm{CK}$, intercellular $\mathrm{CO}_{2}$ concentration $(\mathrm{Ci})$ of the six varieties increased and stomatal
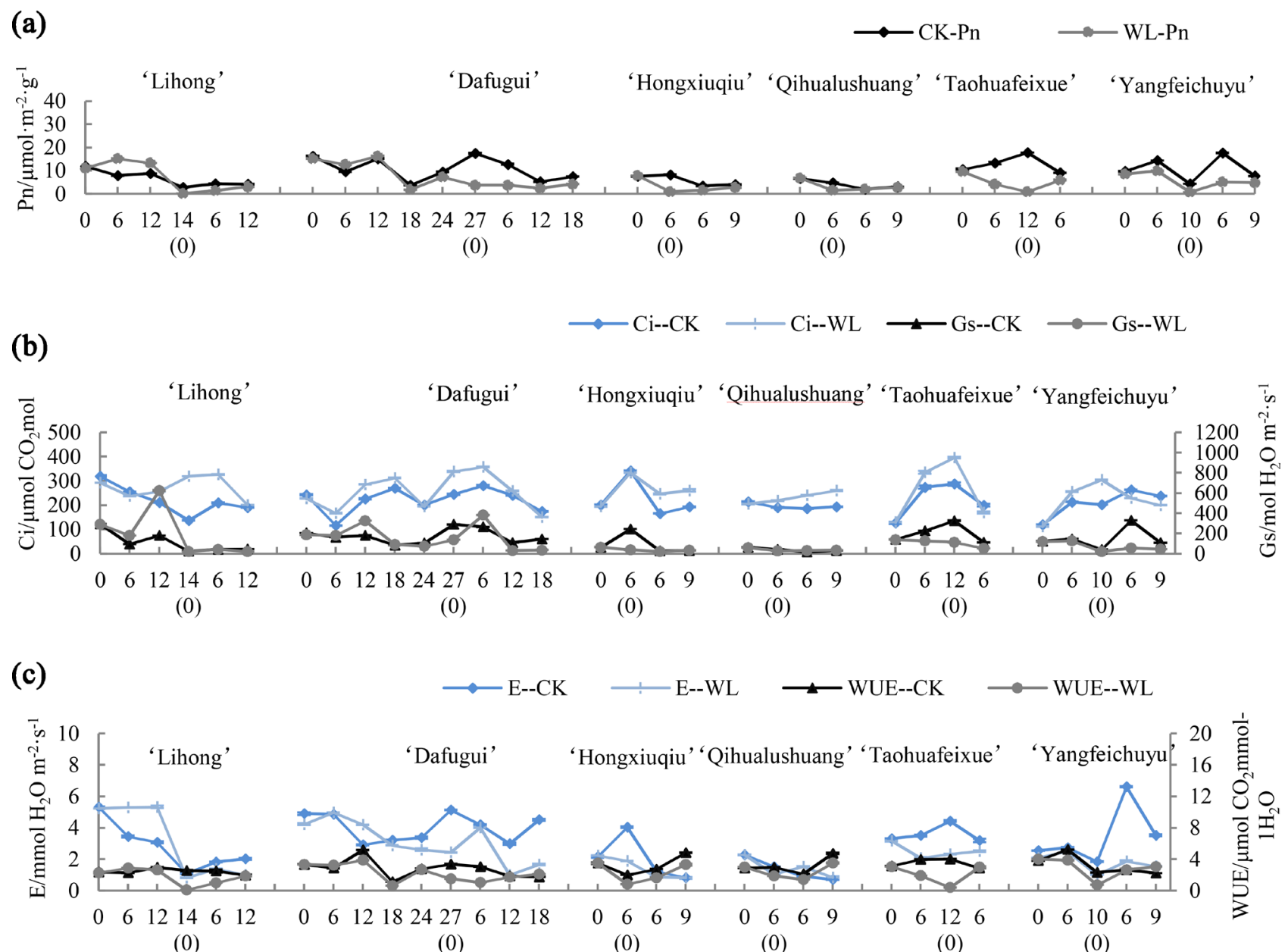

Time (d)

Figure 4. Changes in photosynthetic gas exchange parameters of test materials. (a) the changes of net photosynthetic rate (Pn); (b) the changes of intercellular $\mathrm{CO}_{2}$ concentration $(\mathrm{Ci})$ and stomatal conductance $(\mathrm{Gs})$; (c) the changes of transpiration rate (E) and water utilization rate (WUE). The error bars indicate standard error $(n=3)$. The time of stress removal is marked as the $0^{\text {th }}$ day of the recovery stage. 
conductance (Gs) decreased when stress was removed.

\subsection{Effect of Waterlogging Stress on Chlorophyll Fluorescence Parameters in Six Herbaceous Peony Varieties}

The ratio of variable fluorescence $(\mathrm{Fv})$ to maximum fluorescence $(\mathrm{Fm})(\mathrm{Fv} / \mathrm{Fm})$ represents the maximum photochemical efficiency of PSII and is a reliable indicator of the degree of photoinhibition [15]. When the stress was removed, the Fv/Fm of "Hongxiuqiu" and "Yangfeichuyu" decreased the least compared with $\mathrm{CK}$, indicating a lower degree of photoinhibition. NPQ is the coefficient of heat dissipation. As shown in Figure 5(a), when the stress is removed, the values of "Dafugui", "Taohuafeixue" and "Yangfeichuyu" increase the most than CK (205.66\%, 170.27\%, and 150.05\%), which reflects its strong heat dissipation capacity and can better consume excessive energy through heat dissipation after stress.

qP represents photochemical quenching, which reflects the openness of the PSII reaction center. ETR is the apparent electron transfer rate, and the variation trend of ETR and $\mathrm{qP}$ in this experiment is basically the same. Compared with CK, the ETR of "Qihualushuang" increased the most (110.47\%) and the ETR of "Dafugui" decreased the most (49.77\%) when the stress was removed (Figure 5(b)). When recovering, the ETR of "Lihong" and "Dafugui" decreased, while that
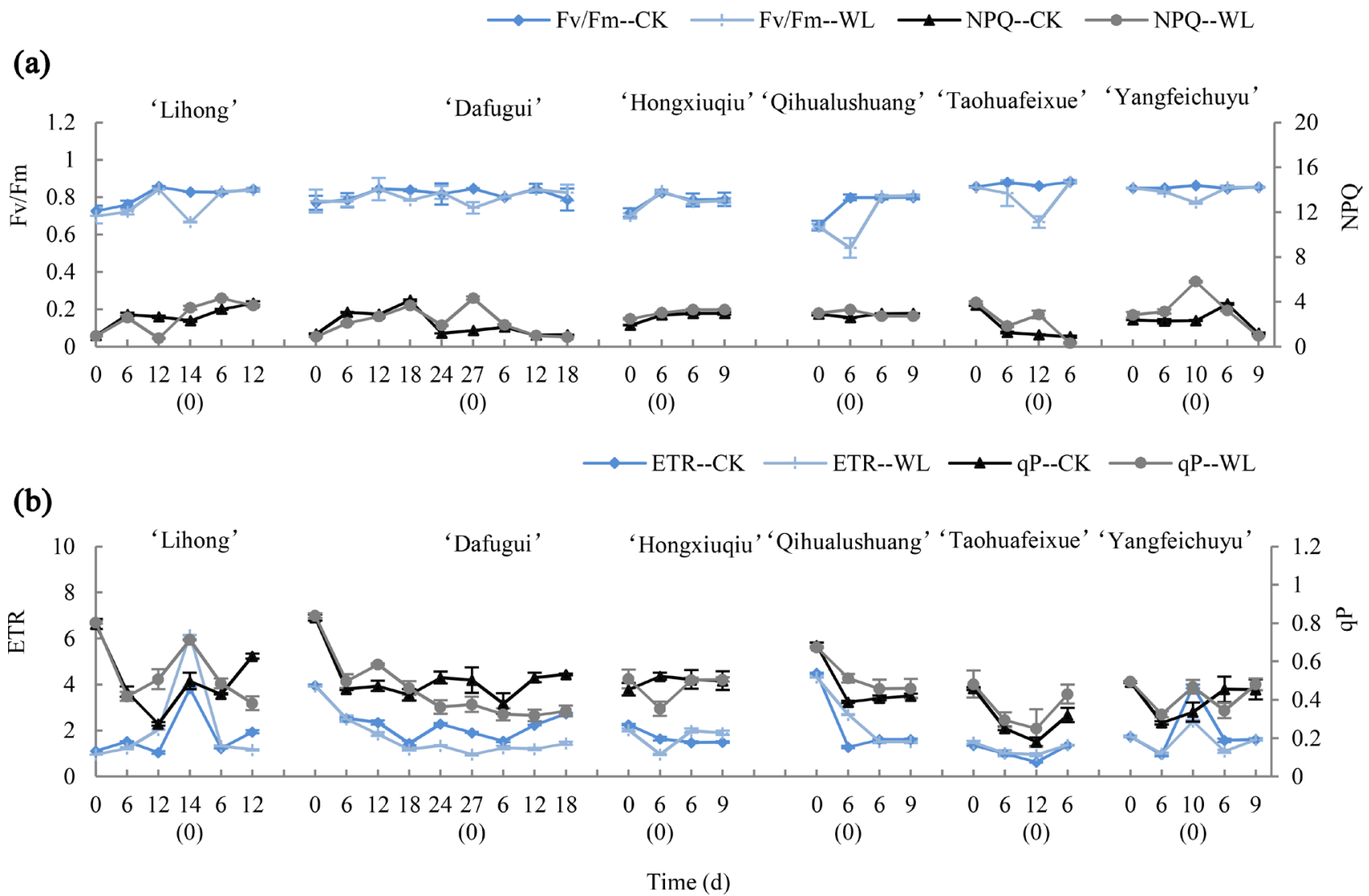

Figure 5. Changes of chlorophyll fluorescence parameters of test materials. (a) the changes of Fv/Fm and NPQ; (b) the changes of ETR and $\mathrm{qP}$. The error bars indicate standard error $(n=3)$. The time of stress removal is marked as the $0^{\text {th }}$ day of the recovery stage. 
of "Hongxiuqiu" increased, and other varieties basically recovered. When the stress was removed, the $\mathrm{qP}$ of "lihong" increased the most (43.28\%), and that of "red hydrangea" decreased the most (32.46\%). When recovering, the qP of "Lihong" and "Dafugui" decreased, and that of "Qihualushuang", "Taohuafeixue", and "Yangfeichuyu" increased.

\subsection{Effects of Waterlogging Stress on the Circulation of Lutein Paeoniflorum in Six Herbaceous Peony Varieties}

Lutein comprises violaxanthin $(\mathrm{V})$, anthe-raxanthin $(\mathrm{A})$, and zeaxanthin $(\mathrm{Z})$. In the xanthophyll cycle, the three components of lutein $(\mathrm{A}, \mathrm{Z}$, and $\mathrm{V})$ are mutually transformed according to changes in light conditions, and both $\mathrm{A}$ and $\mathrm{Z}$ dissipate heat and rely on the xanthophyll cycle. The heat dissipation capacity is positively correlated with the contents of A and Z [16]. As shown in Figure 6, following waterlogging treatment, the $(\mathrm{A}+\mathrm{Z}) /(\mathrm{A}+\mathrm{Z}+\mathrm{V})$ of each variety increased. Compared with CK, the changes in "Taohuafeixue", "Yangfeichuyu", and "Lihong" increased significantly by $140.86 \%, 211.16 \%$, and $90.20 \%$, respectively. It showed that waterlogging stress increased the conversion of $\mathrm{V}$ to $\mathrm{A}$ and $\mathrm{Z}$, and that "Linhong", "Taohuafeixue", and "Yangfeichuyu" can effectively upregulated the lutein cycle to consume excess excitation energy, and thus better protect the light system from damage.

\subsection{Waterlogging Tolerance Coefficient and Principal Component Analysis of Each Individual Index}

Stress resistance in plants is a complex quantitative trait that is affected by many factors. Different varieties have different mechanisms of waterlogging tolerance, and present different responses to specific indicators under adverse conditions [17] [18]. According to the methods and formulas described by Zhou et al. [17], the principal component analysis and membership function analysis were used to comprehensively analyze the tolerance and recovery degree of the tested varieties, and the indexes values of each variety at the $6^{\text {th }}$ day after waterlogging stress and the $6^{\text {th }}$ day after removal stress were selected to calculate and compare

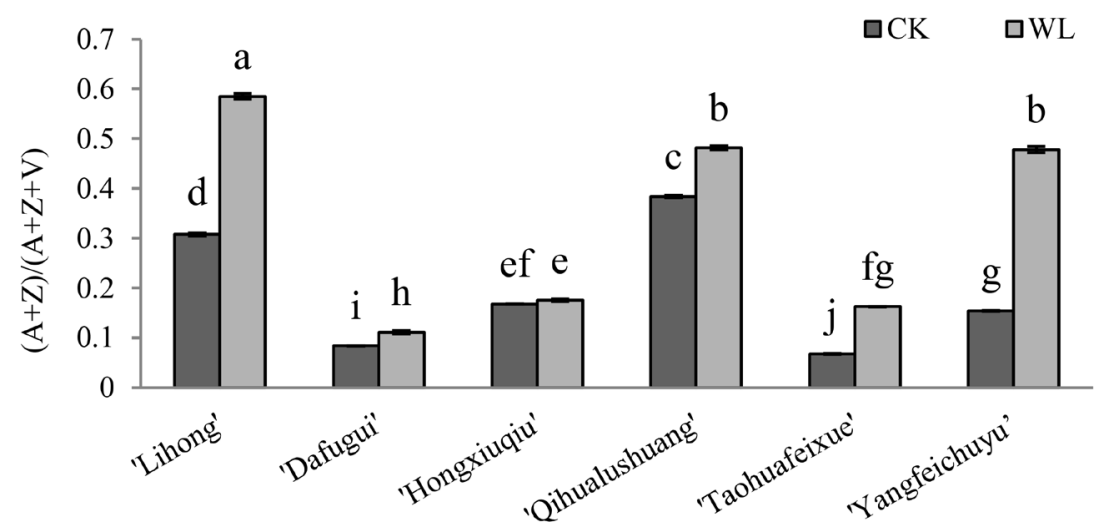

Figure 6. Effects of waterlogging stress on the xanthophyll cycle. The error bars indicate standard error $(n=3)$. 
the waterlogging resistance coefficient and recovery coefficient.

Table 2 shows the different variation ranges of the waterlogging resistance coefficient of each single index of the six varieties. Table 3 is the correlation coefficient matrix of each variety, which shows that there are large or small correlations among all physiological indexes. Through principal component analysis of the waterlogging resistance coefficient of 15 individual indexes, the cumulative contribution rate of the first four comprehensive indexes reached 95.94\% (Table 4 ), and the contribution rate reflected the relative importance of each comprehensive index. According to the index coefficient of each comprehensive index (Table 4) and the waterlogging resistance coefficient of each single index (Table 2 ), the values of the four comprehensive indexes of each variety (Table 5) were calculated.

As shown in Table 5, the waterlogging tolerance $D$ value of each variety was

Table 2. a value of every single index's waterlogging resistant coefficients (\%).

\begin{tabular}{|c|c|c|c|c|c|c|c|c|c|c|c|c|c|c|c|}
\hline Varieties & RWC & REC & ETR & $\mathrm{qP}$ & FvFm & NPQ & Chla & Chlb & Chla $+b$ & Chla/b & Pn & $\mathrm{Ci}$ & Gs & $\mathrm{E}$ & WUE \\
\hline "Lihong" & 96.790 & 132.352 & 80.858 & 94.432 & 94.702 & 90.531 & 108.314 & 104.784 & 107.400 & 103.369 & 192.304 & 93.351 & 189.507 & 153.285 & 125.443 \\
\hline "Dafugui" & 112.931 & 116.842 & 99.788 & 109.451 & 99.023 & 69.426 & 75.531 & 71.189 & 74.375 & 106.100 & 131.250 & 69.489 & 90.418 & 97.746 & 87.791 \\
\hline "Hongxiuqiu" & 102.686 & 119.373 & 58.264 & 67.537 & 101.174 & 107.050 & 84.216 & 95.049 & 86.840 & 88.603 & 10.976 & 97.804 & 15.031 & 46.782 & 41.576 \\
\hline "Qihualushuang" & 101.078 & 103.618 & 210.469 & 131.791 & 66.165 & 127.948 & 69.599 & 81.220 & 72.545 & 85.692 & 31.915 & 114.398 & 65.060 & 69.852 & 63.087 \\
\hline "Taohuafeixue" & 98.320 & 107.712 & 101.862 & 117.639 & 93.660 & 145.411 & 86.365 & 88.135 & 86.809 & 97.992 & 31.281 & 122.777 & 55.066 & 59.087 & 47.859 \\
\hline "Yangfeichuyu" & 104.596 & 136.244 & 103.840 & 115.577 & 97.880 & 138.794 & 79.486 & 83.622 & 80.493 & 95.054 & 68.525 & 120.031 & 86.986 & 91.682 & 74.237 \\
\hline
\end{tabular}

Table 3. Correlation matrix of every single index.

\begin{tabular}{|c|c|c|c|c|c|c|c|c|c|c|c|c|c|c|c|}
\hline Index & RWC & REC & ETR & $\mathrm{qP}$ & $\mathrm{Fv} / \mathrm{Fm}$ & NPQ & Chla & Chlb & Chla + b & Chla/b & Pn & $\mathrm{Ci}$ & Gs & $\mathrm{E}$ & WUE \\
\hline 1 & 1.000 & & & & & & & & & & & & & & \\
\hline 2 & 0.016 & 1.000 & & & & & & & & & & & & & \\
\hline 3 & -0.047 & -0.569 & 1.000 & & & & & & & & & & & & \\
\hline 4 & 0.046 & -0.370 & 0.802 & 1.000 & & & & & & & & & & & \\
\hline 5 & 0.272 & 0.597 & -0.956 & -0.652 & 1.000 & & & & & & & & & & \\
\hline 6 & -0.506 & -0.187 & 0.320 & 0.406 & -0.313 & 1.000 & & & & & & & & & \\
\hline 7 & -0.587 & 0.529 & -0.589 & -0.470 & 0.411 & -0.219 & 1.000 & & & & & & & & \\
\hline 8 & -0.805 & 0.386 & -0.438 & -0.566 & 0.188 & 0.061 & 0.860 & 1.000 & & & & & & & \\
\hline 9 & -0.655 & 0.508 & -0.566 & -0.501 & 0.367 & -0.158 & 0.993 & 0.914 & 1.000 & & & & & & \\
\hline 10 & 0.287 & 0.371 & -0.465 & -0.020 & 0.550 & -0.569 & 0.464 & -0.050 & 0.357 & 1.000 & & & & & \\
\hline 11 & 0.065 & 0.538 & -0.249 & -0.049 & 0.246 & -0.666 & 0.617 & 0.219 & 0.541 & 0.802 & 1.000 & & & & \\
\hline 12 & -0.616 & -0.124 & 0.332 & 0.388 & -0.357 & 0.987 & -0.108 & 0.183 & -0.041 & -0.572 & -0.582 & 1.000 & & & \\
\hline 13 & -0.234 & 0.536 & -0.082 & 0.100 & 0.041 & -0.391 & 0.686 & 0.378 & 0.633 & 0.633 & 0.933 & -0.272 & 1.000 & & \\
\hline 14 & -0.112 & 0.611 & -0.149 & 0.022 & 0.126 & -0.491 & 0.657 & 0.334 & 0.600 & 0.663 & 0.964 & -0.379 & 0.987 & 1.000 & \\
\hline 15 & -0.034 & 0.560 & -0.106 & 0.034 & 0.090 & -0.567 & 0.596 & 0.264 & 0.534 & 0.667 & 0.975 & -0.460 & 0.972 & 0.993 & 1.000 \\
\hline
\end{tabular}


Table 4. Coefficients of comprehensive indexes [CI (x)] and proportion (P).

\begin{tabular}{|c|c|c|c|c|c|c|c|c|c|c|c|c|c|c|c|c|}
\hline Index & RWC & REC & ETR & $\mathrm{qP}$ & FvFm & NPQ & Chla & Chlb & Chla + b & Chla/b & Pn & $\mathrm{Ci}$ & Gs & $\mathrm{E}$ & WUE & $\begin{array}{c}\text { Rate of } \\
\text { Contribution\% }\end{array}$ \\
\hline $\mathrm{CI}(1)$ & 0.025 & 0.149 & 0.058 & 0.202 & -0.005 & 0.031 & 0.038 & -0.067 & 0.016 & 0.169 & 0.180 & 0.041 & 0.211 & 0.206 & 0.196 & 47.278 \\
\hline CI (2) & -0.287 & -0.132 & -0.001 & -0.214 & -0.132 & -0.064 & 0.194 & 0.316 & 0.228 & -0.164 & -0.021 & -0.022 & 0.018 & -0.005 & -0.002 & 22.713 \\
\hline CI (3) & 0.137 & 0.308 & -0.320 & -0.086 & 0.404 & 0.132 & 0.024 & -0.050 & 0.007 & 0.153 & -0.038 & 0.094 & -0.073 & -0.045 & -0.079 & 18.874 \\
\hline CI (4) & -0.069 & 0.303 & -0.024 & 0.299 & 0.134 & 0.456 & -0.010 & -0.069 & -0.023 & 0.076 & -0.049 & 0.443 & 0.061 & 0.039 & -0.019 & 7.074 \\
\hline
\end{tabular}

Table 5. The value of each variety's comprehensive index [CI (x)], indexweight (IW), u (x),Y (x),D, and comprehen-sive evaluation of each variety's waterlogging tolerance (EWT).

\begin{tabular}{|c|c|c|c|c|c|c|c|c|c|c|c|c|c|c|}
\hline Varieties & $C I(1)$ & $C I(2)$ & $C I(3)$ & $C I(4)$ & $u(1)$ & $u(2)$ & $u(3)$ & $u(4)$ & $Y(1)$ & $Y(2)$ & $Y(3)$ & $Y(4)$ & $D$ value & EWT \\
\hline "Lihong" & 1.462 & 1.384 & 0.032 & -0.331 & 1.000 & 1.000 & 0.721 & 0.359 & 0.493 & 0.237 & 0.142 & 0.026 & 0.898 & High \\
\hline "Dafugui" & 0.501 & -1.423 & 0.243 & -1.280 & 0.671 & 0.000 & 0.799 & 0.000 & 0.331 & 0.000 & 0.157 & 0.000 & 0.488 & Medium \\
\hline "Hongxiuqiu" & -1.460 & 0.710 & 0.765 & -0.769 & 0.000 & 0.760 & 0.990 & 0.193 & 0.000 & 0.180 & 0.195 & 0.014 & 0.389 & No \\
\hline "Qihualushuang" & -0.375 & -0.154 & -1.929 & 0.133 & 0.371 & 0.452 & 0.000 & 0.534 & 0.183 & 0.107 & 0.000 & 0.039 & 0.329 & No \\
\hline "Taohuafeixue" & -0.472 & 0.189 & 0.098 & 0.883 & 0.338 & 0.574 & 0.745 & 0.818 & 0.167 & 0.136 & 0.147 & 0.060 & 0.509 & Medium \\
\hline "Yangfeichuyu" & 0.344 & -0.705 & 0.791 & 1.365 & 0.617 & 0.256 & 1.000 & 1.000 & 0.304 & 0.061 & 0.197 & 0.074 & 0.635 & High \\
\hline Weight & & & & & 0.493 & 0.237 & 0.197 & 0.074 & & & & & & \\
\hline
\end{tabular}

ranked as follows: "Lihong" > "Yangfeichuyu" > "Taohuafeixue" > "Dafugui" > "Hongxiuqiu" > "Qihualushuang". The $D$ value for recovery degree of each variety was calculated by the same method, and the degree of recovery after the removal of stress was: "Qihualushuang" > "Lihong" > "Hongxiuqiu" > "Dafugui" > "Yangfeichuyu" > "Taohuafeixue". After a short period of stress (6 days), symptoms of stress appeared; the $D$ value of waterlogging tolerance was the minimum, the recovery process was the fastest and the strongest, indicating that the "Qihualushuang" was the most sensitive to waterlogging stress. The longest duration of stress (27 days) was experienced by "Dafugui", with moderate waterlogging tolerance ( $D$ value) and slow recovery, indicating that "Dafugui" was less sensitive to waterlogging stress; the morphological changes in plants were not obvious in the early stage of stress, but they were physically damaged. The maximum $D$ value of waterlogging resistance, and the rapid recovery of "Lihong" showed that higher water content in the early stage (about 12 days) was beneficial to its growth.

\section{Discussion}

Previous studies have shown that when plant tissues are damaged by adversity, cell membrane structure is damaged or function is impaired, cell membrane permeability increases, leading to increased electrolyte extravasation, which affects plant metabolism [19] [20]. RWC of plant leaves can reflect the degree of water demand, water retention ability and resistance ability, and can reflect the strength of plant vitality and the difference between the degree of water consump- 
tion and recovery ability of plant tissues during transpiration under stress environment [21]. In this experiment, the waterlogging tolerant varieties "Lihong" and "Yangfeichuyu" had higher RWC under stress, and the tested varieties' REC in the leaves at the later stage of waterlogging stress was higher than that of CK, and gradually returned to normal after removing stress, which was consistent with previous research results that stress caused cell membrane damage.

Plant growth requires organic compounds synthesized by photosynthesis in leaves, and Pn is a key indicator that can directly reflect plant growth status. There are two reasons for the decrease of Pn in plants caused by waterlogging stress. First, it is related to the stomatal closure of leaves caused by waterlogging stress. Stomatal closure obstructed the absorption of $\mathrm{CO}_{2}$ by plants and reduced the carbon source of the substrate for photosynthesis, thus reducing the net photosynthetic rate of plants; the other is related to non-stomatal factors [22]. In this experiment, Pn of the tested varieties at the later stage of waterlogging stress was lower than CK, and the decrease of Gs was accompanied by the increase of $\mathrm{Ci}$ and the increase of electrical conductivity, indicating that the main reason for the decrease of Pn in paeoniflora was not the limitation of stoma, but the damage of mesophyll cells. Pn of the waterlogging tolerant variety "Lihong" was significantly higher than CK on the $6^{\text {th }}$ to the $12^{\text {th }}$ day, while pn of the waterlogging tolerant variety lihong was significantly lower than $\mathrm{CK}$ under the stress of "Hongxiuqiu" and "Qihualushuang".

qP reflects the share of light energy absorbed by PSII natural pigments for photochemical electron transfer. A larger $\mathrm{qP}$ indicates a higher electron transfer activity of PSII. Waterlogging stress destroys the internal structure of chloroplasts, significantly reduces $\mathrm{Fv} / \mathrm{Fm}$ and $\varphi$ PSII of plants, and leads to a decrease in Pn. When plants absorb more light energy than they can use, excess light energy leads to photoinhibition or even photodestruction of the photosynthetic apparatus. At this time, plants will adjust the absorption and transmission of light energy through a series of reactions [23] [24] [25]. In this experiment, qP and $\mathrm{Fv} / \mathrm{Fm}$ of herbaceous peony decreased with increasing waterlogging duration, indicating that PSII and electron transport chain activity of all varieties were inhibited under stress. The Fv/Fm of "Qihualushuang" with weak waterlogging resistance and "Taohuafeixue" with medium waterlogging resistance showed a greater decrease. Under stress, qP and ETR of "Lihong" with strong hardy capacity increased, indicating that plants with strong waterlogging tolerance suffered less damage to fluorescence and electron chain transfer activity in leaves.

In terms of the photoprotection mechanism, the initiation of NPQ under stress can prevent excessive light energy transmission to PSII, reduce the over-reduction of PSII and electron transfer chain, and prevent the photooxidation damage of PSII; it is an important indicator of photoprotection, which is not only related to high-energy quenching, but also participates in the protection process of lutein cycle; the lutein cycle is a significant dissipative mechanism, which plays an important role in dissipating excess light energy and protecting photosynthetic mechanism; under the strong light irradiation of photosynthetic organs of plants, 
various components of lutein will undergo $\mathrm{V} \rightarrow \mathrm{A} \rightarrow \mathrm{Z}$ transformation, and previous studies have shown that the contents of $\mathrm{A}$ and $\mathrm{Z}$ are positively correlated with energy dissipation; Guo Shuhua's research on grape showed that the strain with stronger resistance to alkaline salts could more effectively increase xanthophyll cycle to help consume excess light energy, so as to better protect PSII from iinjury [26] [27] [28] [29]. In this test, the NPQ and ( $\mathrm{A}+\mathrm{Z}) /(\mathrm{A}+\mathrm{Z}+\mathrm{V})$ of the tested varieties under waterlogging stress were significantly higher than those of $\mathrm{CK}$, and "Yangfeichuyu" and "Lihong" with higher waterlogging resistance increased more significantly, which could better start NPQ and increase the lutein cycle to protect PSII.

Waterlogged stress caused the growth of hypoxia environment, hinder the plant roots internal mineral transportation, caused the ground part of malnutrition, chlorophyll content decreased, yellow leaves, the waterlogging plants by changing the growth morphology and physiological regulation after the damage to cope with stress, some plants by waterlogging in stress after removing to sprout leaves, show the strong ability of resistance to water logging and resilience [30] [31]. In this experiment, under water logging stress, the chlorophyll content of paeonia lisiflora leaves decreased significantly, and the affected symptoms of redness or yellowing were obvious, but the tolerance time of affected symptoms of $60 \%$ of the leaves of different varieties was different. "Lihong" with strong waterlogging tolerance and "Dafugui" with medium waterlogging tolerance had a longer time to withstand waterlogging stress, with smaller leaf thickness, a larger proportion of leaf tissue density and palisade tissue, and the chlorophyll content of palisade tissue was much higher than that of sponge tissue. But at this point, the chlorophyll content of "Lihong" and "Dafugui" decreases the most, and the chlorophyll cells may be destroyed. The variety "Hongxiuqiu" with weak waterlogging tolerance showed loose arrangement of fence tissue and increased cell space of sponge tissue, which was consistent with the response of apple [32] and tulip to water stress.

In conclusion, the coverage of peony in different varieties of waterlogged stress tolerance, comprehensive waterlogging-resistant ability strong varieties through a relatively favorable morphological structure, maintain high photosynthetic pigment content, at the early stage of the stress by increasing the thermal cycle of consumption, excess light energy dissipation and lutein is less degrees of photoinhibition, maintain higher net photosynthetic rate, longer intimidated to tolerance, when stress damage than plant resistant ability, plants exhibit obvious symptoms, such as red yellow leaves, the varieties with long withstand stress after removing stress also need a long time to recover.

\section{Conclusion}

This experiment analyzed the response and recovery characteristics of paeonia lactiflora to waterlogging stress by simulating waterlogging stress and removing stress. Combined with plant morphology, waterlogging resistance $D$ value and 
recovery degree, it was found that the comprehensive waterlogging tolerance of the 6 tested varieties was as follows: "Lihong" > Yangfeichuyu" > Taohuafeixue" > "Dafugui" > "Qihualushuang" > "Hongxiuqiu". It is suggested that "Lihong" and "Yangfeichuyu" can be cultivated in areas where precipitation concentration is prone to submersible. The screening of waterlogging resistant varieties and the breeding of waterlogging resistant new varieties still need to expand the number of varieties and the screening of waterlogging resistance genes for in-depth research.

\section{Acknowledgements}

This work was supported by the National Natural Science Foundation of China (31670663), and Shandong Agricultural Engineering Forest Tree Breeding Project Corpus for High-grade Garden Flower Breeding (2130106).

\section{Conflicts of Interest}

The authors declare no conflicts of interest regarding the publication of this paper.

\section{References}

[1] Zhang, B.B., Ma, R.J., Cai, Z.X., Shen, Z.J. and Yu, M.L. (2013) Photosynthetic Characteristics Response to Water-Logging in 3 Peach Rootstock Seed-Lings. Acta Botanica Boreali-Occidentalia Sinica, 33, 146-153.

[2] Kumar, P., Pal, M., Joshi, R. and Sairam, K. (2013) Yield, Growth and Physiological Responses of Mung Bean [Vigna radiata (L.) Wilczek] Genotypes to Waterlogging at Vegetative Stage. Physiology and Molecular Biology of Plants, 19, 209-220. https://doi.org/10.1007/s12298-012-0153-3

[3] Pan, L., Xue, Y. and Xue, L. (2011) Advance in Response of Morphology of Plant Waterlogging Stress. Chinese Agricultural Science Bulletin, 27, 11-15.

[4] Chen, X.B. (2013) The Mechanism of Xanthophyll Cycleregulation on Chlorophyll Metabolism of Spinach Leaves under Seawater Stress. Nanjing Agricultural University, Nanjing.

[5] Qin, K.J. (2004) The Herbaceous Peony. China Forestry Press, Beijing, 1-41.

[6] Du, J., Xu, J.G., Sun, X., Lv, M.W., Zhang, Q.X. and Wang, T.L. (2018) Study on Photosynthetic Mechanism of Summer Shading Slowing Aging of Herbaceous Peony (Paeonia lactiflora) Leaves. Plant Physiology, 54, 773-782.

[7] Liu, M., Zhang, Q., Xu, J., Bao, M., Zhang, D., Xie, A. and Sun, X. (2021) Effects of Waterlogging Stress on the Physiological Characteristics and Secondary Metabolites of Herbaceous Peony (Paeonia lactiflora Pall.). American Journal of Plant Sciences, 12, 536-557. https://doi.org/10.4236/ajps.2021.124035

[8] Wang, Q., Liu, J.X., Zhang, J.J. and Yu, X.N. (2014) The Effects of Growth and Physiology of Herbaceous Peony under Water Stress. Journal of Plant Genetic Resources, $15,1270-1277$.

[9] Zhao, S. and Cang, J. (2016) Guidance for Plant Physiology Experiments. China Agriculture Press, Beijing.

[10] Hao, J.J., Kang, Z.L. and Yu, Y. (2007) Plant Physiology Experimental Technology. 
Chemical Industry Press, Beijing.

[11] Robinson, S.A. and Osmond, C.B. (1994) Internal Gradients of Chlorophyll and Carotenoid Pigments in Relation to Photoprotection in Thick Leaves of Plants with Crassulacean Acid Metabolism. Australian Journal of Plant Physiology, 21, 497-506. https://doi.org/10.1071/PP9940497

[12] Lv, C.P., Cheng, M.L., Mo, N.J., Ding, D., Chen, C.T. and Wang, G.K. (2009) Effects of Exogenous GA3 on Flower Bud Development of Herbaceous Peony. Crop Research, 23, 133-137.

[13] Cai, J., Liu, Z.G., Ji, M.C. and Sun, O.W. (2019) Physiological Responses of Two Varieties of Clematis to Flooding Stress. Jiangsu Agricultural Sciences, 47, 154-158.

[14] Kozlowski, T.T. (1997) Responses of Woody Plants to Flooding and Salinity. Tree Physiology Monograph No. 11, 1-29. https://doi.org/10.1093/treephys/17.7.490

[15] Zhao, L.Y., Deng, X.P. and Shan, L. (2005) Effects of Osmotic Stress on Chlorophyll Fluorescence Parameters of Wheat Seedling. Chinese Journal of Applied Ecology, 16, 1261-1264.

[16] Dong, G.F., Chen, Y.Z. and Jiang, Y.M. (1999) Plant Xanthophyll Cycle and Radiationless Energy Dissipation. Plant Physiology Communications, 35, 141-144.

[17] Zhou, G.S., Mei, F.Z., Zhou, Z.Q. and Zhu, X.T. (2003) Comprehensive Evaluation and Forecast on Physiological Indices of Waterlogging Resistance of Different Wheat Varieties. Scientia Agricultura Sinica, 36, 1378-1382.

[18] Hu, H.G., Zhang, Z.M. and Shen, X.H. (2011) Responses of Five Turfgrasse Species to Water Stress and Tolerance Evaluation. Acta Agronomica Sinica, 19, 253-256.

[19] Zhang, Y., Li, R.L., Zhang, D.S., Chen, J.X., Liu, A.Y. and Liu, H.H. (2011) Research Progress on the Effect of Waterlogging on Plants. Crop Research, 25, 420-424.

[20] Liang, K.L., Jia, C.Z., Sun, J.H., Wang, M.Y., Fu, H. and Mao, Z.X. (2019) Evaluation of Physiological Responses and Tolerance to Low-Temperature Stress of Ten Elymus Nutans Varieties. Acta Prataculturae Sinica, 28, 111-121.

[21] Zhang, H.L. and Qi, J.C. (2012) Study on the Relationship between Epicuticular Wax Content of Barley Leaves and Drought Resistance. Xinjiang Agricultural Sciences, 49, 22-27.

[22] Farquhar, G.D. and Sharkey, T.D. (2003) Stomatal Conductance and Photosynthesis. Annual Review of Plant Physiology, 33, 317-345. https://doi.org/10.1146/annurev.pp.33.060182.001533

[23] Reinbothe, S. and Reinbothe, C. (1996) The Regulating of Enzymes Involved in Chlorophyll Biosynthesis. European Journal of Biochemistry, 237, 323-343. https://doi.org/10.1111/j.1432-1033.1996.00323.x

[24] Zhang, S.R. (1999) A Discussion on Chlorophyll Fluorescence Kinetics Parameters and Their Significance. Chinese Bulletin of Botany, 16, 444-448.

[25] Ren B.Z., Zhang, J.W., Dong, S.T., Liu, P. and Zhao, B. (2017) Regulations of 6Benzyladenine (6-BA) on Leaf Ultrastructure and Photosynthetic Characteristics of Waterlogged Summer Maize. Journal of Plant Growth Regulation, 36, 743-754. https://doi.org/10.1007/s00344-017-9677-7

[26] Hunter, N.P., Öquist, G., Hurry, V.M., Krol, M., Falk, S. and Griffith, M. (1993) Photosynthesis, Photoinhibition and Low Temperature Acclimation in Cold Tolerant Plants. Photosynthesis Research, 37, 19-39.

https://doi.org/10.1007/BF02185436

[27] Gilmore, A.M. (1997) Mechanistic Aspects of Xanthophyll Cycle-Dependent Photoprotection in Higher Plant Chloroplasts and Leaves. Physiologia Plantarum, 99, 
197-209. https://doi.org/10.1111/j.1399-3054.1997.tb03449.x

[28] Yamamoto, Y. and Satoh, K. (1998) Competitive Inhibition Analysis of the EnzymeSubstrate Interaction in the Carboxy-Terminal Processing of the Precursor D1 Protein of Photosystem II Reaction Center Using Substituted Oligopeptides. FEBS Letters, 430, 261-265. https://doi.org/10.1016/S0014-5793(98)00671-1

[29] Guo, S.H., Niu, Y.J., Zhai, H., Han, N. and Du, Y.P. (2017) Effect of Alkaline Salt Stress on Fluorescence Characteristics, Xanthophyll Cycle and Cyanide-Resistant Respiration of Grape Hybrid Rootstocks. Plant Physiology, 53, 2013-2021.

[30] Wang, P., Hu, Y.H., Wang, L.M. and Liu, Q.H. (2007) Kinds of Identification Index and the Evaluation Means of Plant Submergence Endurance. Northern Horticulture, 14, 78-81.

[31] Fan, F.F., Yuan, G.W., Li, T.T., Wu, C.P., Zhang, J. and Jiao, J.J. (2018) Effects of Flooding Stress and Drainage on Growth and Physiological Characteristics of Eucalyptus Seedlings. Journal of Zhejiang Forestry Science and Technology, 38, 62-68.

[32] Qu, G.M. and Li, G.X. (1999) Effect of Water Stress on Microstructure of Apple Leaves and New-Born Roots. Acta Horticulturae Sinica, 26, 147-151. 